\title{
Needle Insertion Modelling for the Interactive Simulation of Percutaneous Procedures
}

\author{
S.P. DiMaio and S.E. Salcudean \\ Department of Electrical and Computer Engineering \\ University of British Columbia, Vancouver, Canada \\ \{simond,tims\}@ece.ubc.ca
}

\begin{abstract}
A novel interactive virtual needle insertion simulation is presented. The insertion model simulates three-degree-of-freedom needle motion, physically-based needle forces, linear elastostatic tissue deformation and needle flexibility for the planning and training of percutaneous therapies and procedures. To validate the approach, an experimental system for measuring planar tissue deformation during needle insertions has been developed and is presented. A real-time simulation algorithm allows users to manipulate the virtual needle as it penetrates a tissue model, while experiencing steering torques and lateral needle forces through a planar haptic interface. Efficient numerical computation techniques permit fast simulation of relatively complex two-dimensional and three-dimensional environments at haptic control rates.
\end{abstract}

\section{Introduction}

One of the most common procedures employed in modern clinical practice is the subcutaneous insertion of needles and catheters. In many cases, such procedures are difficult to plan and to perform, and can lead to significant complications if performed incorrectly [1, 2, 3, 4. Physically-based virtual planning and training environments are being developed $[2,5,6,7,8,9,10$; however, the majority of these systems use largely phenomenological and heuristic models that have not been validated, and that are not generalizable. While perhaps effective for the simulation of predominantly 1-DOF problems [6], these approaches may not be suitable for problems involving more complex soft tissue anatomy, needle placement optimisation, trajectory planning and automatic control, where more detailed verifiable knowledge of the biomechanical interaction between surgical needles and soft tissues is required. In prior work, needle insertion forces have been determined for gelatine [11, ex vivo porcine and bovine tissues [5, 12]. In each case, only the resultant force acting at the proximal end of the needle was measured, while in fact penetration forces are distributed along the entire length of the needle axis, resulting from physical phenomena such as cutting/fracture, sliding, friction, stick-slip friction, tissue deformation, tissue displacement and peeling [5]. The needle driving forces measured previously are the integration of this force distribution along the needle shaft. 


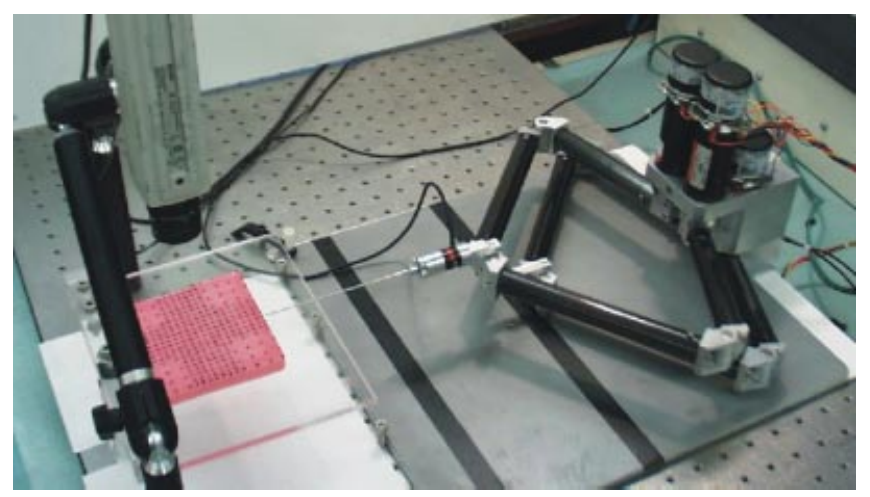

Fig. 1. The complete experimental setup: a robotic manipulator with instrumented epidural needle mounted, a tissue phantom and a CCD camera.

This paper presents a new methodology that has been developed to experimentally determine needle forces during soft tissue puncture, as well as a simulation algorithm for needle insertion mechanics, and is organised as follows. In Section 2, an experimental system for measuring planar tissue phantom deformations during probing and needle insertion is described. Soft tissue modelling and parameterization using a linear elastostatic model is used for estimating the force distribution that occurs along a needle shaft during insertion, as is outlined in Section 3. A numerical simulation of a needle insertion based upon estimated needle force distributions is discussed in Section 4. Issues of real-time performance and haptics are also addressed. Conclusions and discussion of future work are provided in Section 5.

\section{Experimental System to Measure Planar Tissue Deformations}

An experimental setup, shown in Figure 1, has been developed in order to measure the relationship between needle force and 2-D tissue phantom deformation during insertion. A 17 guage Tuohy needle is instrumented with a 6 -DOF force/torque sensor (ATI Nano-17 SI-12-0.12), and is manipulated by a 3-DOF planar device [13. The planar motion of a soft tissue phantom (constructed using a polyvinyl chloride compound) is measured by means of a single CCD camera that is mounted above the needle insertion workspace. Images from this camera are used to track the motion of a set of markings that are applied to the top surface of the phantom, thereby measuring the deformation of the sample.

\section{$3 \quad$ Needle Insertion Force Model}

The relationship between measured insertion force and tissue deformation is characterised by a material model. Forces that occur along the needle shaft are estimated based upon this model, since the direct measurement of needle forces by an instrumentation technique is a challenging problem. If the relationship 


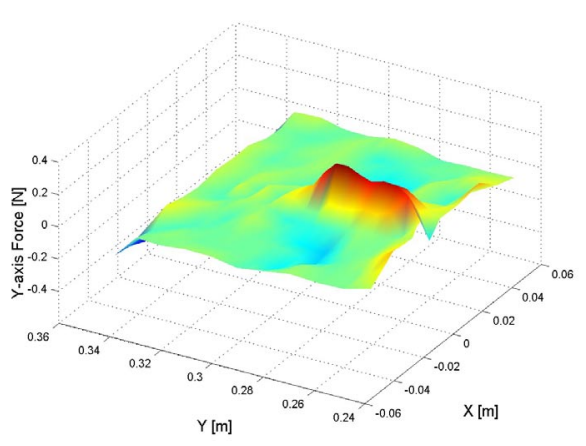

(a)

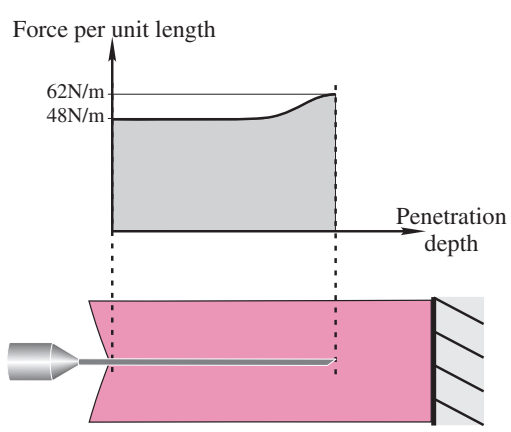

(b)

Fig. 2. (a) Estimated forces at material mesh nodes. (b) Estimated needle force distribution.

between tissue force and displacement is known, then the distribution of force applied along the needle shaft can be computed given only the tissue motion resulting from needle penetration.

Tissue deformation is complex and is still the subject of much research (e.g., [14, 15, 16] and many others). In general, tissue modelling is complex because of inhomogeous, non-linear, anisotropic elastic and viscous behaviour. As a first approximation, this study focuses on linear elastostatic models that are discretised using the Finite Element Method, yielding a set of $2 n$ linear equations that describe tissue deformations in two dimensions:

$$
K_{(2 n \times 2 n)} \underline{u}=\underline{f},
$$

where $\underline{u}$ and $f$ are displacement and force vectors for nodes lying on the mesh discretisation [17. Such models are characterised by two parameters, namely Young's Modulus and the Poisson Ratio, which are identified from boundary probing experiments [18].

An example of tissue phantom forces, derived using experimental measurements and the linear elastostatic material model, is shown in Figure 2(a). Figure 2(b) illustrates the force distribution that is found to occur along the needle during insertion. This distribution is taken from the estimated tissue phantom forces that lie at nodes along the needle, and indicates that axial friction between the needle and the tissue phantom is relatively uniform along the needle shaft. A force peak located immediately behind the needle tip rises approximately $30 \%$ above the friction force, and may be attributable to material cutting. Needle force distributions were determined using experimental measurements taken for a single, fixed needle insertion rate of $1 \mathrm{~mm} / \mathrm{s}$, which is typical in clinical practice.

\section{Needle Insertion Simulation}

Virtual needle insertions are simulated using a numerical material model and the needle shaft force distribution that has been derived. A virtual needle is 

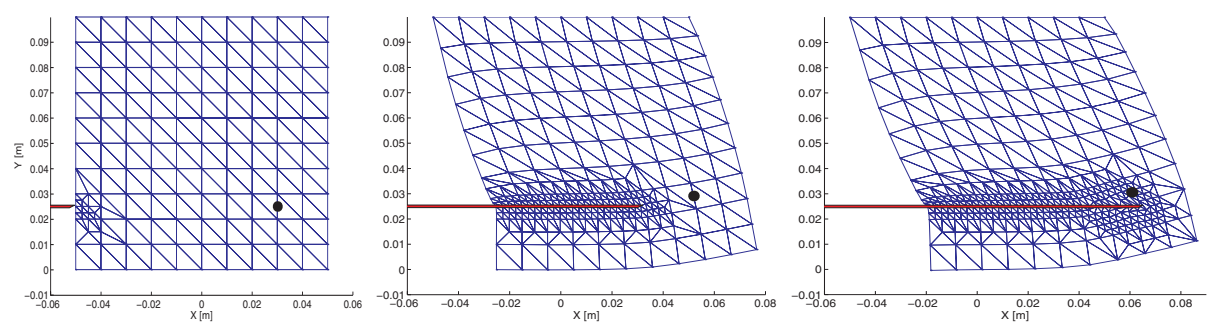

Fig. 3. Simulated needle intercept of a small target embedded within elastic tissue.
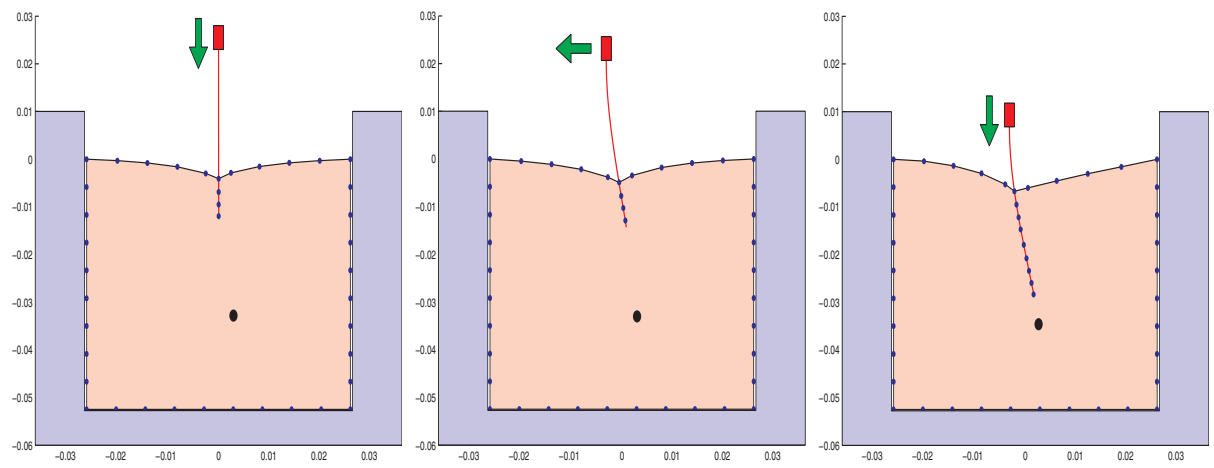

Fig. 4. Simulated needle steering with needle flexibility. Lateral needle motion causes needle flexion, steering the needle toward the target (shown as a disk embedded in the tissue).

advanced into a linear elastostatic model that is discretised using the Finite Element Method [18, while needle shaft forces, distributed as shown in Figure 2b), are applied to model mesh nodes that lie in the path of the needle.

Simulated needle insertions that are based upon the estimated needle forces are shown to reproduce results similar to those observed experimentally. Figure 3 shows a simulated needle insertion into the side of a rectangular tissue model that is rigidly fixed along one edge. The needle axis is initially coincident with a "virtual biopsy target", but fails to intercept the target (shown as a disk), due to tissue deformation. Figure 4 illustrates needle steering (to correct an off-target needle) with a model that includes needle flexibility. Note the nonminimum phase type of response as the base of the needle is moved away from the target, laterally. The potential of physically-based needle insertion simulations for planning and training purposes is thus illustrated.

A real-time implementation of the needle insertion simulator allows users to experience both visual and kinesthetic feedback while executing a virtual planar needle insertion. The haptic simulation system is described in detail in [17]. Realtime computation of needle insertion into soft tissue is complicated by the "curse of dimensionality" that is established by the large number of linear equations required to describe even small models.

The behaviour of a continuum model discretised by the finite element method is observed through the behaviour of a finite set of mesh nodes. For large volumes 


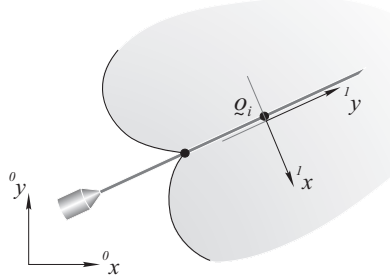

(a)

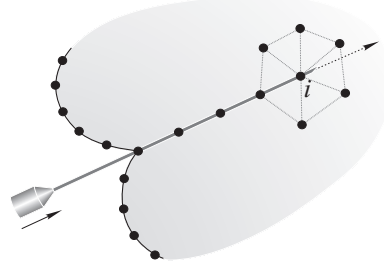

(b)

Fig. 5. (a) Mesh nodes lying along the needle are constrained along the ${ }^{1} x$-axis, and either slip or stick along the ${ }^{1} y$-axis. (b) New intercept nodes are identified by searching within a small neighbourhood centred at the most distal needle node.

of tissue that are finely discretised, the size of matrix $K$ in Equation (11) becomes large due to the large number of material nodes at which force or displacement need to be solved. For needle insertion simulation, it is not necessary to consider the motion of nodes that are not visible (e.g. interior nodes), or the forces applied at nodes that are not in direct contact with the needle shaft. In Figure 3 it is evident that the large majority of mesh nodes are neither visible nor palpable; therefore, the system of linear equations can be reduced to $K_{\mathcal{W}} \underline{u}_{\mathcal{W}}=\underline{f}_{\mathcal{W}}$, where only the behaviour at a small subset $\mathcal{W}$ of mesh nodes (called working nodes) is explicitly considered. At run-time the subset of working nodes $\mathcal{W}$ is selected and the system matrix reduced to $K_{\mathcal{W}}$. As the needle penetrates the tissue surface, it intercepts hidden nodes that need to be re-introduced into the reduced system by simply adding new matrix rows and columns that are derived directly from $K$, which is precomputed. The matrix reduction approach is similar to the condensation techniques discussed in [15], and the Boundary Element Method selected by [14]; however, in this work access to the interior of tissue volumes is retained for quick inclusion when needle penetration occurs.

\subsection{Boundary Conditions and Needle Constraint}

Mesh nodes that are in contact with the needle are constrained by the needle as shown in Figure 5 (a).

If the needle is rigid, then the lateral position of the node is fixed along the ${ }^{1} x$-axis, which constitutes a displacement boundary condition. Along the needle shaft, the node force or node displacement may be constrained, depending upon its state of contact with the needle (i.e. sticking to the needle, or slipping) [18. If the node is free to slide along the needle shaft, then a force boundary condition is applied along the ${ }^{1} y$-axis, and a constant force consistent with the force distribution is applied to the slipping node. If it is in the stuck state, then the node is constrained to lie at a fixed point on the needle, along the ${ }^{1} y$-axis.

The system of equations in $K_{\mathcal{W}}$ is rearranged in order to reflect the resulting inhomogeneous collection of boundary conditions:

$$
K_{\mathcal{W}} \underline{u}_{\mathcal{W}}=\underline{f}_{\mathcal{W}} \rightarrow K_{\mathcal{W}}^{\prime} \underline{\mathbf{x}}_{\mathcal{W}}=\underline{\mathbf{y}}_{\mathcal{W}}
$$

where $\underline{x}_{\mathcal{W}}$ and $\underline{y}_{\mathcal{W}}$ are formed by exchanging elements between $\underline{u}_{\mathcal{W}}$ and $\underline{f}_{\mathcal{W}}$. 
Needle node boundary conditions change frequently during simulation, depending upon the commanded motion of the needle. A single boundary condition change can be expressed as an inexpensive low rank update:

$$
\left(K_{\mathcal{W}}^{-1}\right)^{\prime}=K_{\mathcal{W}}^{-1}-\frac{\mathbf{c}_{i} \cdot \mathbf{r}_{i}}{p_{i}}
$$

where $p_{i}$ is the $i^{\text {th }}$ pivot of $K_{\mathcal{W}}^{-1} ; \mathbf{c}_{i}$ and $\mathbf{r}_{i}$ are the $i^{\text {th }}$ column and $i^{\text {th }}$ row of $K_{\mathcal{W}}^{-1}$, with the exception of their $i^{t h}$ coordinates, which are set to $\left(p_{i}+1\right)$ and $\left(p_{i}-1\right)$, respectively. $\left(K_{\mathcal{W}}^{-1}\right)^{\prime}$ is the new system matrix and vectors $\underline{x}_{\mathcal{W}}$ and $\underline{y}_{\mathcal{W}}$ must be adjusted accordingly (i.e. by exchanging displacement and force variables). This approach to boundary condition changes results in an $O\left(N^{2}\right)$ computation rather than the $O\left(N^{3}\right)$ operation required to re-invert stiffness matrix $K_{\mathcal{W}}$, in a way that is similar to the capacitance matrix strategy presented by James and Pai in [14].

\subsection{Local Coordinate Changes}

The coordinate system shown in Figure 5(a) is fixed to the needle; therefore, as its orientation changes it is necessary to effect local coordinate changes in $K_{\mathcal{W}}^{-1}$. When the boundary conditions are uniform, this results in a simple affine transformation:

$$
{ }^{0} u_{\mathcal{W}}=K_{\mathcal{W}}^{-10} f_{\mathcal{W}} \Rightarrow{ }^{1} u_{\mathcal{W}}=A^{T} K_{\mathcal{W}}^{-1} A^{1} f_{\mathcal{W}}
$$

where ${ }^{0} u_{\mathcal{W}}$ and ${ }^{0} f_{\mathcal{W}}$ are displacement and force vectors in a nominal system coordinate frame, while ${ }^{1} u_{\mathcal{W}}$ and ${ }^{1} f_{\mathcal{W}}$ are the vectors after rotating the coordinate frame at the $i^{t h}$ node by an angle $\theta$. Matrix $A$ is composed of $(2 \times 2)$ rotation submatrices on its diagonal [18].

If node $i$ has different boundary conditions along its two coordinate axes, then such a tranformation is not possible, due to the mixed force and displacement variables in $\underline{x}_{\mathcal{W}}$ and $\underline{y}_{\mathcal{W}}$. The local coordinate transformation for a node that is sliding along the needle axis has the following form:

$$
\underline{\mathbf{x}}_{\mathcal{W}}=\left(M-K_{\mathcal{W}}^{-1} N\right)^{-1}\left(K_{\mathcal{W}}^{-1} M-N\right){ }^{1} \underline{\mathbf{y}}_{\mathcal{W}}
$$

where $M$ and $N$ are sparse transformation matrices. Due to the properties of $M$ and $N,\left(M-K_{\mathcal{W}}^{-1} N\right)$ is shown to be inexpensively inverted, and the new system computed [18]. Node coordinate frames must be updated incrementally from one simulation sample period to the next, according to the change in needle orientation angle $\Delta \theta$. If the needle is curved or flexible, then the local coordinate system transformations will vary along the length of the needle shaft.

\subsection{System Solution}

The reduced system matrix $K_{\mathcal{W}}^{-1}$ evolves from sample period to sample period, due to boundary condition and local coordinate system updates, and is used 

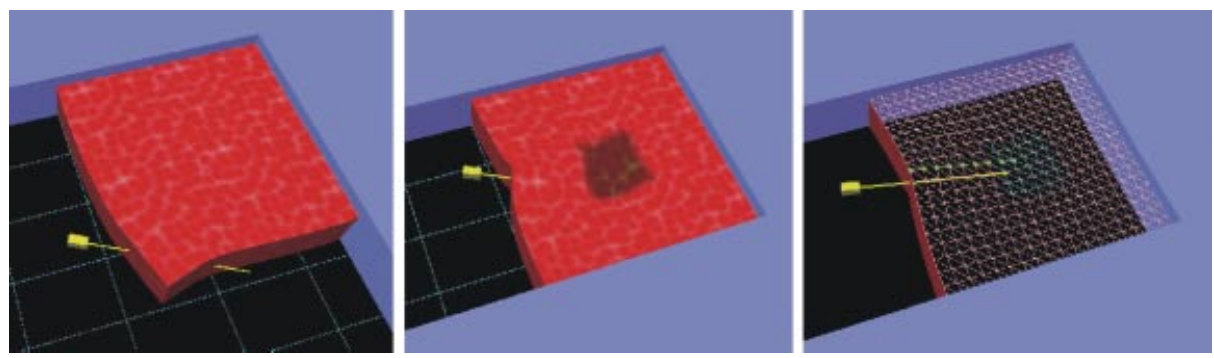

Fig. 6. Interactive virtual needle insertion in a planar environment.

to solve for $\underline{\mathbf{x}}_{\mathcal{W}}$, the vector of unknown node forces and displacements. Node positions are updated for graphical rendering of the scene and needle node forces are integrated for feedback via the haptic interface. The interactive real-time model shown in Figure [6] consists of 361 nodes and is computed at a rate of $500 \mathrm{~Hz}$ by a $450 \mathrm{MHz}$ P-III PC, without any particular effort to optimise code.

\section{Conclusion}

This paper presents a system for interactively simulating virtual needle insertions that is based upon experimentally determined needle insertion mechanics. A novel approach for estimating needle shaft forces and tissue behaviour using a measurement system and soft tissue deformation models has been developed. It is based on the established Finite Element Method and parameters identified during experiments.

The haptically-enabled virtual insertion environment allows users to manipulate a three-degree-of-freedom needle as it penetrates a discretised inhomogeneous linear elastostatic tissue model. Unlike existing single axis simulation, steering torques and lateral needle forces can be felt, while tissue model deformation is observed. Real-time simulation of this model is challenging due to the large system of equations involved, as well as frequent topological and boundary condition changes that occur as the needle moves into the tissue model. We have developed a fast algorithm for interactive needle insertion with force feedback, without loss of model detail or degradation in global response. The haptic simulation, described in [17], achieves a sample rate of $500 \mathrm{~Hz}$ for a $2-\mathrm{D}$ virtual tissue model and planar haptic interface, using a desktop PC. While it was developed for modelling 2D tissue phantoms, the method can be generalised to 3D.

Needle mechanics measurements and simulations are of interest for the development of physically-based virtual planning and training systems that are aimed at reducing the incidence of complications in clinical practice. Current and future work will explore 3-D modelling techniques, further biomechanics experiments, non-linear material models, the effects of material inhomogeneities and dynamics (including needle feed rate dependence), as well as model-based planning and control of needle insertion procedures. 


\section{References}

1. Datta, S.: Complications of regional analgesia and anaesthesia. In: Proceedings of the $17^{\text {th }}$ Annual European Society of Regional Anaesthesia Congress. (1998)

2. Azar, F.S., Metaxas, D.N., Schnall, M.D.: A Finite Element Model of the Breast for Predicting Mechanical Deformations during Biopsy Procedures. In: Proc. of the IEEE Workshop on Math. Methods in Biomedical Image Analysis. (2000) 38-45

3. Nath, S., Chen, Z., Yue, N., Trumpore, S., Peschel, R.: Dosimetric effects of needle divergence in prostate seed implant using ${ }^{125} \mathrm{I}$ and ${ }^{103} \mathrm{Pd}$ radioactive seeds. In: Medical Physics, American Institute of Physics (2000) 1058-1066

4. Fiducane, B.: Complications of brachial plexus anaesthesia. In: Proceedings of the $17^{\text {th }}$ Annual European Society of Regional Anaesthesia Congress. (1998)

5. Brett, P.N., Parker, T.J., Harrison, A.J., Thomas, T.A., Carr, A.: Simulation of resistance forces acting on surgical needles. In: Proceedings of the Inst. of Mech. Engineers. Part H, Journal of Engineering in Medicine. Volume 211. (1997) 335-347

6. Hiemenz, L., McDonald, D.J., Stredney, D., Sessanna, D.: A Physiologically Valid Simulator for Training Residents to Perform an Epidural Block. In: Proceedings of the $15^{\text {th }}$ Southern Biomedical Engineering Conference. (1996)

7. Kwon, D.S., Kyung, J.U., Kwon, S.M., Ra, J.B., Park, H.W., Kang, H.S., Zeng, J., Cleary, K.R.: Realistic Force Reflection in a Spine Biopsy Simulator. In: Proc. of the IEEE Int. Conf. on Robotics and Automation. (2001) 1358-1363

8. Shimoga, K.B., Khosla, P.K.: Visual and Force feedback to Aid Neurosurgical Probe Insertion. In: Proceedings of the $16^{\text {th }}$ Annual International Conference of the IEEE Engineering in Medicine and Biology Society. Engineering Advances: New Opportunities for Biomedical Engineers. Volume 2. (1994) 1051-1052

9. Miller, S., Jeffrey, C., Bews, J., Kinsner, W.: Advances in the Virtual Reality Interstitial BrachyTherapy System. In: Proceedings of the Canadian Conference on Electrical and Computer Engineering. (1999) 349-354

10. Zeng, J., Kaplan, C., Bauer, J., Xuan, J., Sesterhenn, I.A., Lynch, J.H., Freedman, M.T., Mun, S.K.: Optimizing prostate needle biopsy through 3-D simulation. In: Proceedings of SPIE Medical Imaging. (1998)

11. Hiemenz, L., Litsky, A., Schmalbrock, P.: Puncture Mechanics for the Insertion of an Epidural Needle. In: Proceedings of the Twenty-First Annual Meeting of the American Society of Biomechanics. (1997)

12. Simone, C., Okamura, A.: Haptic Modeling of Needle Insertion for Robot-Assisted Percutaneous Therapy. In: IEEE Int. Conf. on Robotics and Automation. (2002)

13. Sirouspour, M.R., DiMaio, S.P., Salcudean, S.E., Abolmaesumi, P., Jones, C.: Haptic Interface Control - Design Issues and Experiments with a Planar Device. In: Proceedings of the IEEE Int. Conf. on Robotics and Automation. (1999)

14. James, D.L., Pai, D.K.: ArtDefo, Accurate Real Time Deformable Objects. In: Computer Graphics - Proceedings of SIGGRAPH '99. (1999)

15. Bro-Nielsen, M.: Finite Element Modeling in Surgery Simulation. In: Proceedings of the IEEE. Volume 86. (1998) 490-503

16. Hagemann, A., Rohr, K., Stiehl, H.S., Spetzger, U., Gilsbach, J.M.: Nonrigid Matching of Tomographic Images Based on a Biomechanical Model of the Human Head. In: Medical Imaging 1999 - Image Processing (MI '99). (1999) 583-592

17. DiMaio, S.P., Salcudean, S.E.: Simulated Interactive Needle Insertion. In: Proc. of the $10^{t h}$ Symposium on Haptic Interfaces for Virtual Environments and Teleoperator Systems, IEEE Virtual Reality. (2002)

18. DiMaio, S.P., Salcudean, S.E.: Needle Insertion Modelling and Simulation. In: IEEE International Conference on Robotics and Automation. (2002) 